\title{
Simulation of Internal Solitary Waves with Negative Polarity in Slowly Varying Medium
}

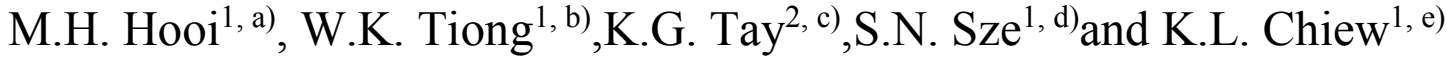 \\ ${ }^{1}$ Faculty of Computer Science and Information Technology, Universiti Malaysia Sarawak, 94300 Kota Samarahan, \\ Sarawak, Malaysia. \\ ${ }^{2}$ Department of Communication Engineering. Faculty of Electric and Electronic, UniversitiTun Hussein Onn \\ Malaysia, 86400 Parit Raja, Johor, Malaysia. \\ a)reyonhooi@gmail.com \\ b)Corresponding author: wktiong@unimas.my \\ c)tay@uthm.edu.my \\ d)snsze@unimas.my \\ e)klchiew@unimas.my
}

\begin{abstract}
We consider the propagation of an internal solitary wave over two different types of varying depth regions, i.e. a gentle monotonic bottom slope connecting two regions of constant depth in two-layer fluid flow and a smooth bump. Here, we let the depth of the upper layer is smaller than the lower layer such that an internal solitary wave of negative polarity is generated. The appropriate model for this problem is the variable-coefficient extended Korteweg-de Vries equation, which is then solved numerically using the method of lines. Our numerical results show different types of transformation of the internal solitary wave when it propagates over the varying depth region depending on the depth of the lower layer after the varying depth region including generation of solitary wavetrain, adiabatic and non-adiabatic transformation of the internal solitary wave.
\end{abstract}

\section{INTRODUCTION}

Internal solitary waves (ISWs) exist within a fluid medium in the interior of density stratified fluid system. In the past few decades, ISWs have been observed instratified fjords [1], straits [2] andare mostly found in layered ocean especially on the continental slope [3]-[7] and in the marginal regions[8]-[9].Several studies, for instance [10]-[12], have been conducted on ISWs to figure out the properties of the ISWs propagation. Huang et. al. [13] shows that the ISWs can potentially have large amplitude and strong currents during propagation. Due the nonlinearity characteristic and dispersion behaviour of ISWs, they can propagate over a long distance horizontally on the denser layer and retain their wave structure[8]. Meanwhile, they may carry enormous momentum as they travel over long distancesuch that they could affect the ocean activities[14], e.g. supply of nutrients for photosynthesis, transportation of sediments and pollutants, acoustic transmission, submarine navigation [13] and the underwater man-made buildings such as deepsea drilling platform [15]. Therefore,studies on internal waves become a valuable research to understand the behaviour of ISWs.

TheKorteweg-de Vries (KdV) equation[16]is widely used for the description of weakly nonlinear internal wave in shallow water. Itwas used by Benney[17] and Benjamin [18] to model the ISWs and followed by many others researchers [19]-[25]. For certain conditions in stratified fluids, the nonlinearity of ISWs mainly comes from the cubic nonlinear term because they often have a very small or zero coefficient in nonlinear term with large amplitude propagating across the continental shelf area [26]-[27].Therefore, a cubic nonlinear term is added into the KdV equation to describe the structure of ISWs [20]. Thus, the KdV equation is replaced by the extended Korteweg de Vries (eKdV) equation, which is commonly known as the Gardner equation. Stanton and Ostrovsky[28] shows that 